\title{
Automated Podosome Identification and Characterization in Fluorescence Microscopy Images
}

\author{
Marjolein B.M. Meddens, ${ }^{1}$ Bernd Rieger, ${ }^{2}$ Carl G. Figdor, ${ }^{1}$ Alessandra Cambi, ${ }^{1,3, *}$ and \\ Koen van den Dries ${ }^{1}$ \\ ${ }^{1}$ Department of Tumor Immunology, Nijmegen Centre for Molecular Life Sciences, Radboud University \\ Nijmegen Medical Centre, P.O. Box 9101, 6500 HB Nijmegen, The Netherlands \\ ${ }^{2}$ Quantitative Imaging Group, Department of Imaging Science \& Technology, Delft University of Technology, \\ Lorentzweg 1, 2628 CJ Delft, The Netherlands \\ ${ }^{3}$ Nanobiophysics, MIRA Institute for Biomedical Technology and Technical Medicine and MESA+ Institute for \\ Nanotechnology, Faculty of Science and Technology, University of Twente, P.O. Box 217, 7500 AE Enschede, \\ The Netherlands
}

\begin{abstract}
Podosomes are cellular adhesion structures involved in matrix degradation and invasion that comprise an actin core and a ring of cytoskeletal adaptor proteins. They are most often identified by staining with phalloidin, which binds F-actin and therefore visualizes the core. However, not only podosomes, but also many other cytoskeletal structures contain actin, which makes podosome segmentation by automated image processing difficult. Here, we have developed a quantitative image analysis algorithm that is optimized to identify podosome cores within a typical sample stained with phalloidin. By sequential local and global thresholding, our analysis identifies up to $76 \%$ of podosome cores excluding other F-actin-based structures. Based on the overlap in podosome identifications and quantification of podosome numbers, our algorithm performs equally well compared to three experts. Using our algorithm we show effects of actin polymerization and myosin II inhibition on the actin intensity in both podosome core and associated actin network. Furthermore, by expanding the core segmentations, we reveal a previously unappreciated differential distribution of cytoskeletal adaptor proteins within the podosome ring. These applications illustrate that our algorithm is a valuable tool for rapid and accurate large-scale analysis of podosomes to increase our understanding of these characteristic adhesion structures.
\end{abstract}

Key words: podosomes, image analysis, fluorescence microscopy, actin, image quantification, cytoskeletal adaptor proteins

\section{INTRODUCTION}

Podosomes are highly dynamic adhesion structures that are involved in matrix degradation and invasion (Linder, 2007; Gawden-Bone et al., 2010). Structurally, they comprise a dense actin core, which has a diameter of approximately $350 \mathrm{~nm}$, surrounded by a $250 \mathrm{~nm}$ wide ring of integrins and integrin-associated proteins (Block et al., 2008). With lifetimes between 2-12 min, podosomes are very dynamic and continuously change size, shape, and composition (Destaing et al., 2003; Evans et al., 2003). They have initially been identified in Src-transformed fibroblasts, and although previously thought to be restricted to only a few cell types, they have now been observed in smooth muscle cells, activated endothelial cells, and cells of the myeloid lineage such as osteoclasts, macrophages, and dendritic cells (DCs) (Murphy \& Courtneidge, 2011). More recently, podosome-associated structures named invadopodia have been found on the ventral membranes of tumor cells where they are thought to play a central role in tumor metastasis (Blouw et al., 2008; Mukhopadhyay et al., 2009).

Most of our current understanding of podosome formation, turnover, and distribution is based on studies that

Received June 27, 2012; accepted September 9, 2012

*Corresponding author. E-mail: a.cambi@ncmls.ru.nl exploit conventional and confocal microscopy techniques. Although these techniques are potentially quantitative, largescale image analysis is required to obtain enough statistical power to be able to detect subtle and transient variations in podosome composition. Since manual identification of podosomes is very time-consuming ( $>1 \mathrm{~h}$ for a typical image) and requires sufficient experience from the researcher, a reliable automated detection method is an essential tool to study the mechanisms that regulate podosome organization and behavior.

Podosomes are most often identified by a filamentous actin (F-actin) staining, which also serves as a reference for the co-staining with other core or ring components. The F-actin staining is commonly performed with fluorochromeconjugated phalloidin, which gives a typical punctate pattern within fluorescence images, which is potentially well suited for automated detection. In recent years extensive progress has been made in methods for detection of spots in fluorescence microscopy images, which could also be applied to detect podosome cores. These methods range from morphological image filtering (Kimori et al., 2010), waveletbased multiscale detectors (Olivo-Marin, 2002), to machine learning methods (Jiang et al., 2007). Also in the particle tracking field, many different algorithms exist that localize and subsequently track subdiffraction sized particles also in 
A

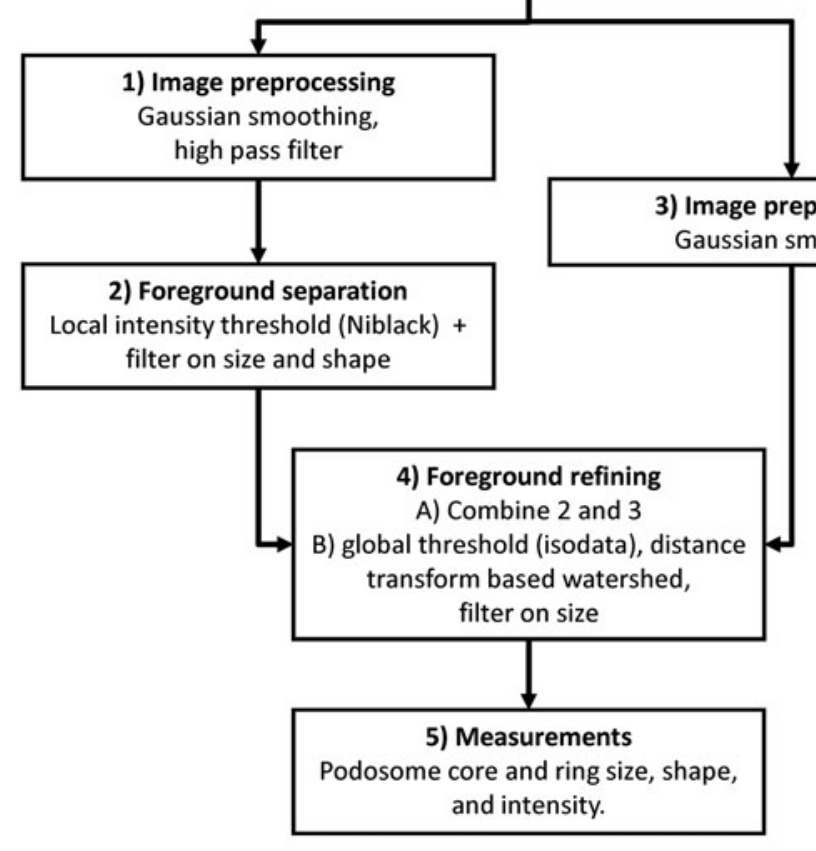

B
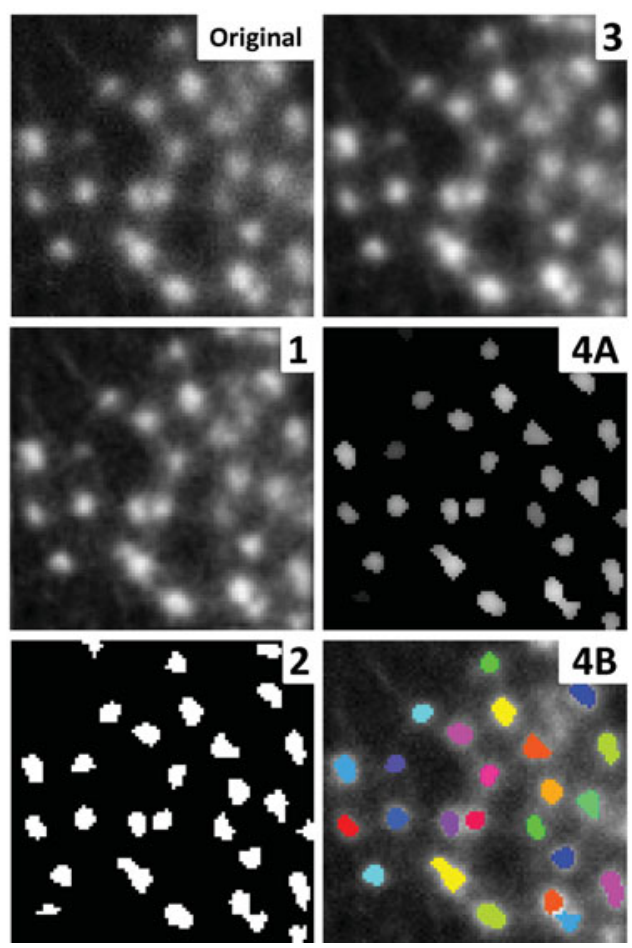

Figure 1. Schematic overview of algorithm. (A) Main processing steps of algorithm. (B) Segmentation of podosomes; the original image is preprocessed using Gaussian filtering and an unsharp mask (1) or Gaussian filtering only (3), a Niblack intensity threshold on (1) separates the foreground to produce a mask (2), which is combined with image (3) to produce image (4A), an isodata threshold, distance transform based watershed operation and filter on shape and size result in the regions of interest, which are represented as labeled objects in (4B).

the presence of background fluorescence (Kalaidzidis, 2007; Smal et al., 2010). However, several difficulties arise when applying these methods for the detection of podosome cores in phalloidin-stained images. First, although essential for proper identification of podosomes, phalloidin does not specifically stain the F-actin within podosome cores. Phalloidin also binds F-actin in cellular ruffles, focal adhesions, and other F-actin rich structures that are visualized within a typical sample. Second, the intensity of the podosome cores within a typical image is highly heterogeneous. While some podosome cores may be brighter than most of the background structures, others are much dimmer. These issues result in a high number of false positives and false negatives, respectively, when established spot-detection methods for the identification of podosome cores in fluorescent images are applied. This urges the need for a specific analysis algorithm to identify podosome cores within a phalloidinstained sample.

We therefore developed a quantitative image analysis algorithm that was optimized to identify podosome cores within a typical sample stained with fluorochromeconjugated phalloidin. By sequential local and global thresholding, our analysis readily identifies up to $76 \%$ of podosome cores excluding other F-actin-based structures. Moreover, we demonstrate that based on the overlap in podosome identifications and quantification of podosome numbers, our algorithm performs equally well compared to three experts. Finally, we show that our analysis provides accurate quantitative information on podosome core and ring composition and structure, illustrating that it can be a valuable tool for the large-scale analysis of podosomes to increase our understanding of these small adhesion structures.

\section{Materials and Methods}

\section{Description of the Algorithm}

The basic idea behind the algorithm is to separate podosome F-actin cores from other F-actin rich cellular structures within phalloidin-stained samples based on intensity, shape, and size. To achieve a good foreground separation, we have developed a method that combines local and global thresholding, after suitable preprocessing to suppress noise. The algorithm is divided into six steps (Figs. 1A, 1B). To allow distinction between podosomes and other small F-actin rich cellular structures, the image is preprocessed in two different ways (steps 1 and 3). The first preprocessing is optimized for local thresholding to segment the foreground (step 2), which results in a mask of all the structures that stand out from the background in the original image. The second preprocessing step is optimized to prevent the artificial enhancement of small dim cellular structures. Then, the resulting image from step 3 is combined with the mask obtained in step 2 to allow global thresholding (step $4 \mathrm{~A})$. The global threshold applied to the combined image is calculated via the isodata method (Ridler \& Calvard, 1978). After the global threshold, the image is segmented by a 
watershed algorithm, and subsequently podosome cores are selected based on size and shape (step 4B). The information about the localization of the core can be used to calculate the position of the ring that surrounds the core, after which various podosome characteristics, such as the structure and composition of the core and ring, can be determined (step 5).

\section{Step 1. Image Preprocessing for Foreground Separation}

To enable rough podosome segmentation, the original image is preprocessed using Gaussian smoothing to reduce noise and a high pass filter to enhance the contrast of small cellular features. For Gaussian smoothing, a standard deviation of $\sigma=0.5$ pixel $=50 \mathrm{~nm}$ was chosen to reduce noise as well as to enhance object visibility, without affecting the structure and intensity profile of the podosome cores within the image. Second, unsharp masking is applied by subtracting the Laplacian of the image from the image itself. The width of the Laplace operator is essential to filter for the right object sizes and should be about 10 times smaller than the expected diameter of the podosomes.

\section{Step 2. Foreground Separation}

To separate the foreground, the image is thresholded by on a local threshold operation (Niblack, 1986) given by

$$
I_{B}>\mu_{B}+\frac{1}{2} \sigma_{B}
$$

where $I$ are the pixel intensities, and $\mu$ the average and $\sigma$ the standard deviation of the pixel intensities in a circular structuring element $B$. The diameter of the structuring element is set to be about 1.3 times larger than the expected diameter of the podosomes. The first segmentation, which is created by the Niblack threshold, does not yet allow the selection of podosomes based on shape, size, or intensity due to the fact that some of the other F-actin rich cellular structures, which are still present, have roughly the same brightness, size, and shape as podosomes. Therefore, after labeling the segmentation, only a rough selection of the objects is applied to remove the outliers, based on the area (number of pixels) and roundness of the objects. Roundness is calculated using

$$
R=\frac{4 A}{\pi d^{2}}
$$

where $A$ is the number of foreground pixels and $d$ is the maximal Feret diameter, which is the width of the longest projection (Feret, 1931). For an ellipse the roundness becomes the aspect ratio. The selection criteria for the area and roundness depend on the expected average and variation in size of the podosomes, which are specified for each application. The boundaries used here are 1.5 times smaller and larger than the lower and upper expected size limits of podosomes, respectively. The lower and upper limits for the roundness are set to 0.4 and 1 . The resulting segmentation mask will then be combined with the image that will be obtained in step 3 of the algorithm.

\section{Step 3. Image Preprocessing for Foreground Refining}

The first image preprocessing step does not only enhance podosomes but also other small cellular features that are present in the original image. The mask obtained in step 2 therefore still contains many unwanted features that should not be identified as podosome cores. To allow the separation of the podosome cores from the other objects, the first mask is combined with a Gaussian smoothed version of the original image. For Gaussian smoothing, a standard deviation sigma of 1 pixel $(100 \mathrm{~nm})$ was chosen to filter out objects much smaller than podosome cores while leaving podosome sized objects intact.

\section{Step 4. Foreground Refining}

To segment the podosomes a global threshold is applied. For this, an automated isodata threshold is used (Ridler \& Calvard, 1978) in which all background pixels that result from the masking are ignored. The global threshold is followed by a watershed segmentation on the distance transform of the binary image (Vincent \& Soille, 1991) to separate connected/touching blob like objects. The final selection of podosome cores is made from this mask based on size.

\section{Step 5. Measurements}

The segmented podosomes can be used to quantitatively investigate podosome core intensity, size, and shape. To study the structure and intensity of the podosome rings, the individual segmentations can be easily manipulated (dilated or eroded). We can calculate the intensity in the ring around the podosomes as a function of distance from the initial segmentation mask. To this end we create a binary mask $M_{r}$ as

$$
M_{r}=r_{\min }<D T(o b j)<r_{\max }
$$

where $D T$ is the distance transform on the logical complement of the podosome segmentations, $r_{\min }$ and $r_{\max }$ are the minimal and maximal distance from the segmentation boundary, and obj are the segmented podosome cores. Subsequently, fluorescence intensity of F-actin, or costainings of other proteins, can be determined by applying these segmentation masks. For our application it was sufficient to use a radius step of 1 pixel between subsequent $r_{\min }$ and $r_{\max }$, but subpixel steps are also possible.

\section{Preparation of Human DCs}

DCs were generated from monocytes, which were isolated from peripheral blood mononuclear cells as described previously (Thurner et al., 1999; de Vries et al., 2002). Monocytes were derived either from buffy coats or leukapheresis products. Plastic-adherent monocytes were cultured for 6 days in RPMI 1640 medium (Life Technologies, Carlsbad, CA, USA) supplemented with $10 \%$ fetal bovine serum (Greiner Bio-One, Kremsmünster, Austria), $1 \mathrm{mM}$ Ultraglutamine (BioWhittaker, Inc., Walkersville, MD, USA), antibiotics (100 U/mL penicillin, $100 \mu \mathrm{g} / \mathrm{mL}$ streptomycin, and $0.25 \mu \mathrm{g} / \mathrm{mL}$ amphotericin B; Gibco, Grand Island, NY, 
USA), IL-4 $(500 \mathrm{U} / \mathrm{mL})$, and GM-CSF $(800 \mathrm{U} / \mathrm{mL})$ in a humidified, $5 \% \mathrm{CO}_{2}$ containing atmosphere.

\section{Antibodies and Reagents}

The following antibodies were used: mouse anti-vinculin, mouse anti-talin (both Sigma-Aldrich), mouse anti-paxillin (BD Transduction Laboratories, Franklin Lakes, NJ, USA), and goat anti-zyxin (Santa Cruz Biotechnology, Inc., Santa Cruz, CA, USA). Texas Red-conjugated phalloidin (Invitrogen Corporation, Carlsbad, CA, USA) was used to stain F-actin. The following compounds were used: $2.5 \mu \mathrm{g} / \mathrm{mL}$ Cytochalasin D, $20 \mu \mathrm{M}$ Blebbistatin (both Sigma-Aldrich, St. Louis, MO, USA) and $10 \mu \mathrm{M}$ prostaglandin $\mathrm{E}_{2}\left(\mathrm{PGE}_{2}\right.$, Cayman Chemical Company, Ann Arbor, MI, USA).

\section{Immunofluorescence}

Cells were seeded on glass coverslips, left to adhere for $4 \mathrm{~h}$, and stimulated or left untreated. The cells were fixed in $3.7 \%(\mathrm{w} / \mathrm{v})$ formaldehyde in phosphate buffered saline (PBS) for $10 \mathrm{~min}$. Cells were permeabilized in $0.1 \%(\mathrm{v} / \mathrm{v})$ Triton X-100 in PBS for 5 min and blocked with 2\% (w/v) bovine serum albumin in PBS. The cells were incubated with primary $\mathrm{Ab}$ for $1 \mathrm{~h}$. Subsequently, the cells were washed with PBS and incubated with Alexa Fluor 488labeled secondary Abs for 45 min. Finally, cells were incubated with Texas Red-conjugated phalloidin for $30 \mathrm{~min}$ and washed with phosphate buffer prior to embedding in Mowiol (Sigma-Aldrich). The cells were imaged with a Leica DMRA fluorescence microscope (Leica Microsystems, Wetzlar, Germany) with a $63 \times$ PL APO $1.3 \mathrm{NA}$ oil immersion objective and a Leica DFC340 FX charge-coupled device camera with $1200 \times 1600,8$ bit pixels. Samples were excited with an OSRAM HBO103 w/2 mercury lamp and a green fluorescent protein (GFP) and N2.1 filtercube were used to image the Alexa Fluor 488-labeled secondary Abs and Texas Redconjugated phalloidin, respectively. The GFP and N2.1 channels were sequentially acquired with an integration time of $200 \mathrm{~ms}$ and $10 \mathrm{~ms}$, respectively. Imaging was also performed on a Olympus FV1000 confocal laser scanning microscope (Olympus, Tokyo, Japan) with a $60 \times 1.35 \mathrm{NA}$ oil immersion objective, Texas Red-conjugated phalloidin was excited with a $559 \mathrm{~nm}$ diode laser and emission fluorescence was filtered by a $575-675 \mathrm{~nm}$ band-pass filter and detected through a $125 \mathrm{~nm}$ pinhole. Frames were acquired at $1200 \times 1600$ pixels with $100 \mathrm{~nm}$ pixel size, 16 bits per pixel, and $12.5 \mu$ s pixel time.

\section{Transfection}

Transient transfection of LifeAct-GFP (gift of Michael Sixt, Max Planck Institute of Biochemistry, Martinsried, Germany) was carried out with the Neon Transfection System (Invitrogen). Cells were washed with PBS and resuspended in $115 \mu \mathrm{L}$ resuspension buffer per $0.5 \times 10^{6}$ cells. Subsequently, cells were mixed with $5 \mu \mathrm{g}$ DNA per $10^{6}$ cells per transfection and electroporated. Directly after, cells were transferred to WillCo-dishes (WillCo Wells bv., Amsterdam, The Netherlands) with prewarmed medium without antibiotics or serum. After $3 \mathrm{~h}$, medium was replaced by medium supplemented with $10 \%(\mathrm{v} / \mathrm{v})$ fetal calf serum and antibiotics.

\section{Live Cell Imaging}

Prior to live cell imaging, cells were washed with PBS and imaging was performed in RPMI without phenol red supplemented with $25 \mu \mathrm{M}$ HEPES. Transiently transfected cells were imaged on a Zeiss LSM 510 microscope (Carl Zeiss, Oberkochen, Germany) equipped with a PL APO $63 \times / 1.4$ NA oil immersion objective. GFP was excited with a $488 \mathrm{~nm}$ argon laser and detected through a $125 \mathrm{~nm}$ pinhole. Images (140 nm pixel size) were acquired every $15 \mathrm{~s}$ at $37^{\circ} \mathrm{C}$ with a $12.5 \mu$ s pixel time.

\section{Statistical Analysis}

Statistical analysis was carried out with GraphPad Prism and Microsoft Excel. Data are presented as mean for line graphs and median \pm interquartile range for box plots. A Student's $t$-test was used for comparison of two groups. Statistical significance was defined as $P<0.05$.

\section{Image Processing and Software}

The algorithm described in this work is available online at http://www.diplib.org/add-ons. All image processing and analysis were implemented using MATLAB (version 2012a, 64 bit) and the DIPimage (v2.4) toolbox.

\section{Results}

\section{Performance of the Algorithm}

To assess the performance of the algorithm we collected a representative wide-field microscopy image of DCs, seeded onto glass coverslips, and subsequently stained with Texas Red-conjugated phalloidin and a monoclonal anti-vinculin antibody (Supplementary Fig. 1).

\section{Supplementary Material}

To view Supplementary Material for this article, please visit http://dx.doi.org/10.1017/S1431927612014018.

Figure 2A displays a cropped region of the F-actin image, clearly revealing the difficulties that arise for the automated segmentation of podosome cores. First, the F-actin content of podosome cores differed a lot between podosomes; therefore, some podosomes appeared much brighter than the background, whereas others were only just above background intensity, which made the automatic identification difficult. Second, not all small bright objects within the image were podosomes. Many objects within the image remained difficult to evaluate, even by an experienced researcher. We assessed the performance of the algorithm by comparing its outcome with podosome identifications by experienced researchers. All three experts, who have extensive experience in the interpretation of podosome images, used the vinculin co-staining along with the F-actin staining to identify podosomes within the image 
A

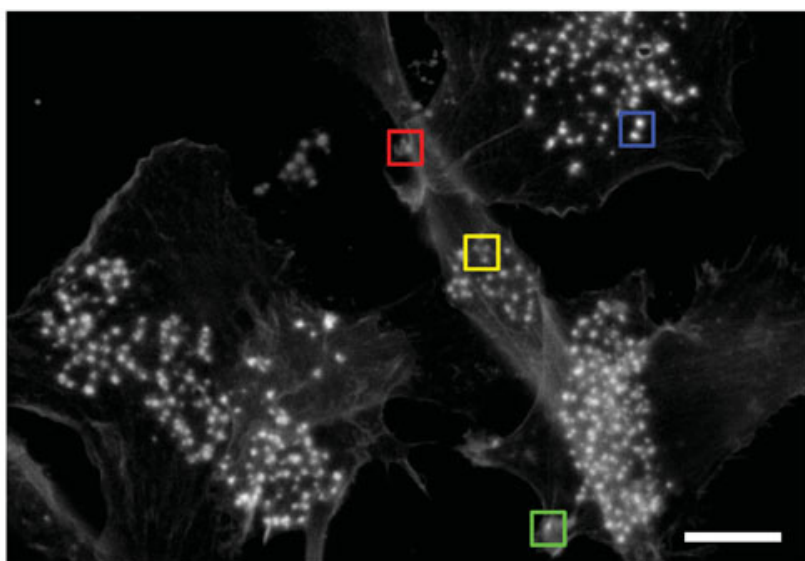

Bright podosomes Dim podosomes
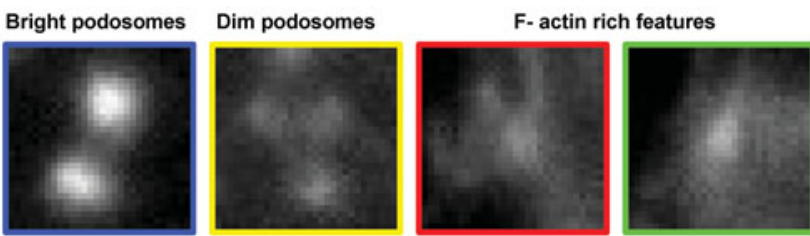

B

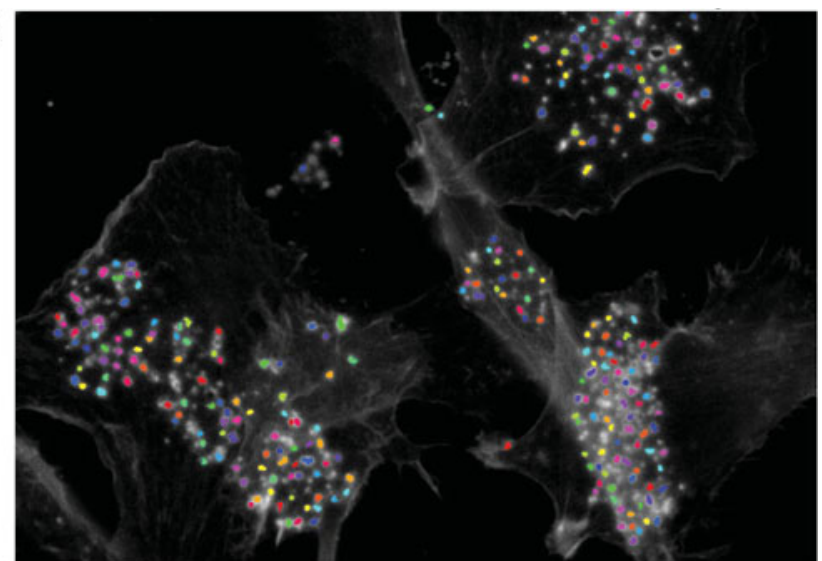

C

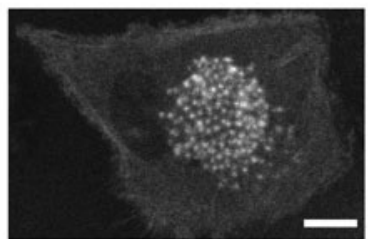

D

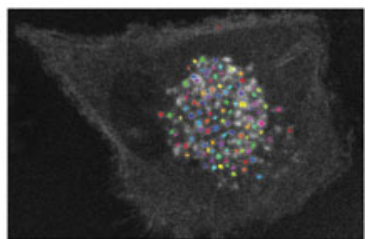

Figure 2. Segmentation of podosomes in images of fixed and live cells. DCs were seeded on glass coverslips, fixed and stained with Texas Red-conjugated phalloidin and imaged in widefield (A); insets show podosomes and other F-actin rich features. DCs were electroporated with LifeAct-GFP, seeded on glass Willco dishes, and live cell imaging was performed on a confocal microscope (C). The algorithm was applied to the images in $\mathbf{A}$ and $\mathbf{C}$; the output is shown as labeled podosome core segmentations in B and D. Scale bars represent $10 \mu \mathrm{m}$.

shown in Supplementary Figure 1. The experts selected (by mouse click) each object they identified as a podosome core, and the coordinates of these positions were saved. Next, the algorithm was applied to the F-actin image only. An object segmented by the algorithm was considered to be the same as an expert's identification when the coordinates of the expert's position were within a 3 pixel radius of the object. Table 1 shows the overlap between experts and the algorithm. Although the algorithm detected fewer podosomes than the experts, the overlap among experts was comparable to that of the algorithm. This demonstrates that the algorithm can be considered a solid tool for the detection of podosomes within phalloidin stained samples.

To assess the performance of the algorithm in images with fewer F-actin rich structures besides podosome cores, we manually cropped the image in Supplementary Figure 1 around podosome clusters. This resulted in 17 cropped images of podosome clusters on which we then applied the algorithm to identify the podosome cores. Again, we compared the segmented areas to the expert's identifications. An object was considered to be a podosome core when at least two expert's positions were within a 3 pixel radius of the segmented area. Cropping the image increased the sensitivity of podosome core detection from $73 \%$ to $76 \%$ while the false positives decreased from $16 \%$ to $5 \%$ (Table 2), indicating that the performance of the algorithm is even better in images with isolated podosome clusters.

To study the dynamics of podosomes, live cell imaging is mostly carried out on cells transfected with a fluorescent protein linked to either G-actin or an F-actin probe. To assess the performance of the algorithm in this type of image, we performed confocal microscopy on DCs electroporated with LifeAct-GFP, which is an F-actin specific probe. In the resulting time-lapse movies, only one cell was visible per image. The performance of the algorithm was assessed in the same way as described above for three independent LifeAct-GFP images, of which one is shown in Figure 2C. The output of the algorithm for the image in Figure $2 \mathrm{C}$ is shown in Figure 2D. The average sensitivity of the algorithm on the three images was $76 \%$ with $5 \%$ false positives. The performance of the algorithm in live cell confocal microscopy was comparable to the performance in fixed,

Table 1. Performance of the Algorithm on Image in Supplementary Figure 1A.

\begin{tabular}{lccccc}
\hline & $\begin{array}{c}\text { Number of } \\
\text { Podosomes } \\
\text { Identified }\end{array}$ & $\begin{array}{c}\text { Overlap with } \\
\text { Expert 1 }\end{array}$ & $\begin{array}{c}\text { Overlap with } \\
\text { Expert 2 }\end{array}$ & $\begin{array}{c}\text { Overlap with } \\
\text { Expert 3 }\end{array}$ & $\begin{array}{c}\text { Overlap with } \\
\text { Algorithm }\end{array}$ \\
\hline Expert 1 & 1,217 & - & 1,066 & 893 & 901 \\
Expert 2 & 1,236 & 1,066 & - & 938 & 906 \\
Expert 3 & 1,301 & 893 & 938 & - & 881 \\
Algorithm & 1,070 & 901 & 906 & 881 & - \\
\hline
\end{tabular}


Table 2. Performance of the Algorithm Compared to Combined Identifications of Three Experts.

\begin{tabular}{lcc}
\hline & & $\%$ False \\
& Sensitivity & Positives \\
\hline Large image & $73 \%$ & $16 \%$ \\
Podosome cluster crop & $76 \%$ & $5 \%$ \\
Life-Act-GFP movie & $73 \%$ & $17 \%$ \\
\hline
\end{tabular}

wide-field microscopy, showing the applicability of the algorithm to study podosome dynamics in live cell movies.

\section{Quantification of Podosome Numbers}

Podosome numbers vary greatly between different cells and conditions. To facilitate fast and reliable quantification of podosome numbers, automated detection is required. We assessed the ability of the algorithm to quantify differences in podosome numbers. Therefore, we performed a time course of $\mathrm{PGE}_{2}$ stimulation, which is known to induce rapid podosome dissolution in DCs (van Helden et al., 2006, 2008). DCs were seeded on glass coverslips and either left untreated or incubated for up to 10 min with $10 \mu \mathrm{M} \mathrm{PGE}_{2}$, before being fixed and stained for F-actin and vinculin. For each condition five images containing multiple cells were taken on a wide-field fluorescence microscope. To avoid over- or underestimation of the number of podosomes per cell, only cells that were completely visible within an image were analyzed. This resulted in at least 21 cells per condition that were analyzed. The outcome of the algorithm was compared with podosome counts from experienced researchers. The experts used the vinculin co-staining along with the F-actin staining to count the number of podosomes per cell, whereas the algorithm was applied to the F-actin image only.

Visual inspection of the images showed a time dependent decrease in the number of podosomes per cell (Fig. 3A), which was confirmed by the manual quantification of the three experts (Fig. 3B, dashed lines). The average number of podosomes per cell decreased from 178 before $\mathrm{PGE}_{2}$ stimulation to 17 after 10 min $\mathrm{PGE}_{2}$ stimulation. The results that were obtained with the algorithm were very similar to the expert counts with only a small underestimation observed for the first three time points. This demonstrates the applicability of our algorithm to quantify podosome numbers with high accuracy, in a fast, reliable way.

\section{Actin Density in Podosome Core and Network}

Podosomes consist of dense actin cores, which are surrounded by actin filaments that are associated with myosin II (Gawden-Bone et al., 2010). These actin filaments are collectively called the actin network (Luxenburg et al., 2007), and since this network cannot be resolved by conventional microscopy, it appears as a cloud that surrounds the actin cores within low resolution images (Destaing et al., 2003). To further evaluate our image analysis algorithm, we have tested whether we could assess changes in the F-actin intensity in the core and the surrounding network upon myosin II inhibition by blebbistatin (blebb) and inhibition of actin polymerization by cytochalasin D (cytoD), two frequently used compounds to study actin-based adhesion structures. For this purpose, DCs were seeded onto glass
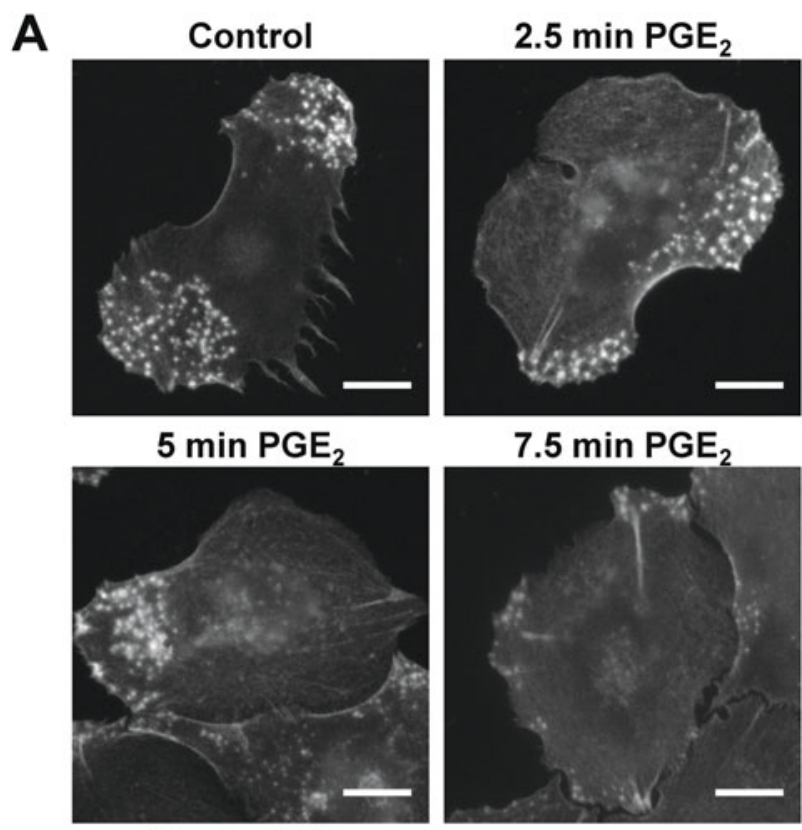

$10 \mathrm{~min} \mathrm{PGE}_{2}$
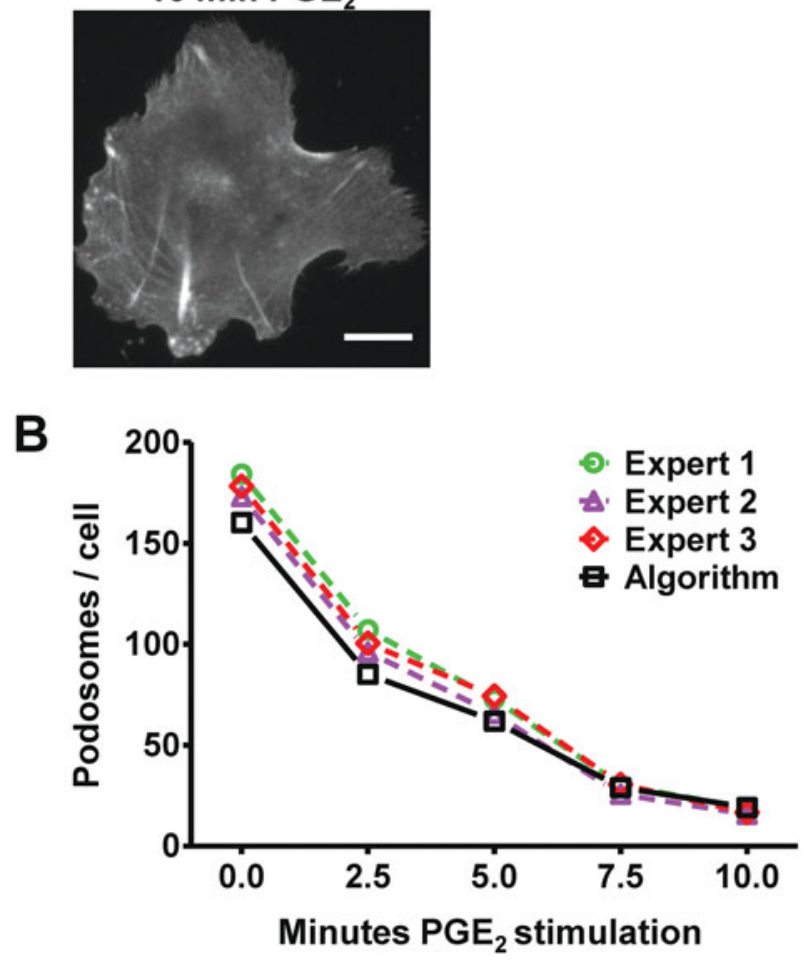

Figure 3. Quantification of podosome numbers. DCs were seeded on glass coverslips; cells were left untreated or were treated for the indicated times with $10 \mu \mathrm{M} \mathrm{PGE}$. Afterward, the cells were fixed and labeled with Texas Red-conjugated phalloidin (A). Podosome numbers were quantified for at least 21 cells per condition, and the average number of podosomes per cell is shown in $\mathbf{B}$. The dashed lines show manual counts of three experts, and the solid line shows the quantification by the algorithm. Scale bars represent $10 \mu \mathrm{m}$. 
coverslips and were either left untreated or were incubated with $20 \mu \mathrm{M}$ blebb or $10 \mu \mathrm{M}$ cytoD, before being fixed and stained for F-actin. For each condition at least five images, containing multiple cells, were taken on a confocal microscope, allowing the comparison of over 800 podosomes per condition. The F-actin intensity in the core was determined by measuring the mean fluorescence intensity for each segmented podosome in the original image. To determine the F-actin intensity in the actin network, a segmentation mask was created to select the network area for each podosome. The distance transform was used to select pixels closer than four pixels distance to the edge of the nearest segmentation outside the core and closer than one pixel distance inside the core segmentation. By comparison to the distance transform of each individual segmentation, each pixel was assigned to a specific podosome core. The resulting segmentation masks were subsequently used to calculate the mean fluorescence intensity for actin in the network. By visual inspection of the images, no clear differences between treated and untreated samples could be observed. Instead, the large-scale analysis clearly showed a statistically significant increase in F-actin intensity in the core and the surrounding network upon myosin II inhibition (Figs. 4A, $4 \mathrm{~B})$. On the contrary, inhibition of actin polymerization decreased the F-actin intensity around the core as well as in the core itself. These results show the applicability of automated assessment of individual podosomes to determine intensity levels of podosome components, with a statistical power not afforded before.

\section{Podosome Ring Composition}

To further exploit the use of our image analysis algorithm, we set out to measure the distribution of podosome ring components with respect to the podosome core. To determine the intensity of ring components, cells were stained for both F-actin and a ring component (Fig. 4C, upper left panel). Podosome cores were segmented in the F-actin image (upper middle and right panels) and a distance transform was calculated for both the segmentation mask and its logical complement. From these distance transforms masks were created for pixels at each distance from the boundary of the segmented area, both within the segmented area (lower left panel) and in the background (lower right panel). These masks were subsequently used to measure the average pixel intensities in the co-staining of the ring components (Fig. 4). For each condition five images, together containing at least 1,120 podosomes, were measured.

Cytoskeletal adaptor proteins, such as vinculin, talin, paxillin, and zyxin, are abundantly present within podosomes rings. They are thought to link the integrins in the ring with the actin in the podosome core. Although these four proteins are generally being referred to as ring components, their localization around the podosome core varies greatly. For example, vinculin appears as a distinct ring, whereas talin appears to form a "carpet" that contains holes at the sites where the podosome cores are located (Fig. 4D). Using our analysis we quantitatively assessed the structural differences between the localization of the four adaptor proteins. Therefore, we seeded DCs onto glass coverslips, fixed and stained them with Texas Red-conjugated phalloidin and specific antibodies to visualize F-actin and the ring components vinculin, talin, paxillin, and zyxin, respectively. Subsequently, we identified the podosome cores within these images and created segmentation masks as described above. The mean fluorescence intensity within each masked area was measured for at least five images per condition. In this way the localization of the adaptor proteins with respect to the core was determined. Figure $4 \mathrm{E}$ shows that whereas vinculin and paxillin both formed a ring with a diameter larger than the core diameter, talin was more homogeneously distributed between podosome cores and zyxin formed a ring within the diameter of the core. These results show the applicability of the podosome core detection algorithm to quantitatively assess ring component distribution. The differential distribution of the cytoskeletal adaptor proteins in podosome rings most likely correlates with their many specific interaction partners and their function in podosome biology.

\section{Discussion}

In this study we describe, for the first time, a procedure for automated detection of podosome cores in fluorescence (confocal) microscopy images. This method combines local and global thresholding on differentially filtered versions of the original image and can be used to quantitatively detect and measure intensities of podosome core and ring components.

Podosomes are most often identified by a staining of F-actin; however, F-actin is not only present in podosome cores, but also in many other cellular components such as focal adhesions and stress fibers. So far, no specific podosome core or ring marker has been identified. Therefore, an algorithm to identify podosomes should be able to detect podosome cores by other means than based solely on the intensity of a certain fluorescently labeled protein. In our algorithm we combine intensity, by local and global thresholding, with size and shape of the objects to discriminate podosomes from other F-actin rich cellular features. Alternatively, rather than an F-actin alone, a co-staining with a ring component could be used to increase the sensitivity and specificity of podosome segmentation. However, we have shown in Figure 4E that ring components are differentially distributed around the podosome core. A podosome detection algorithm based on the staining of a ring component would therefore oblige the user to always measure the same ring marker, possibly requiring a triple staining for each experiment. Moreover, the localization of ring components can vary between experimental conditions (Luxenburg et al., 2012) making the staining of a ring component unreliable in experiments aimed at detecting changes in podosome composition and structure. Therefore, we based and optimized our algorithm on the F-actin staining only, to ensure a broad applicability for the investigation of all aspects of podosome biology. 
A
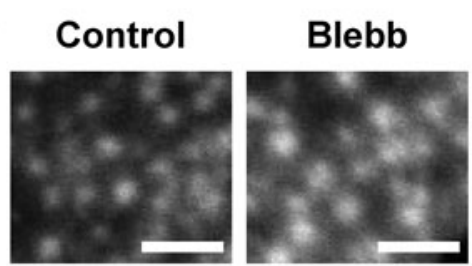

CytoD

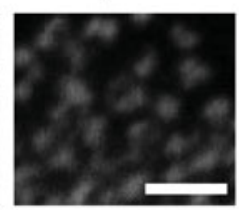

B

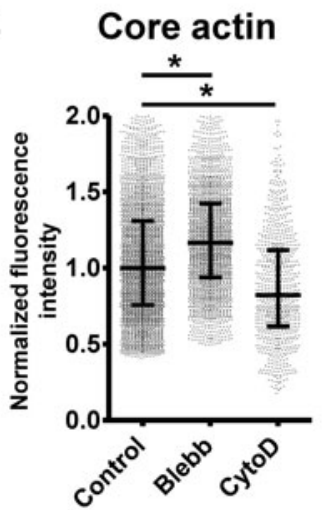

C

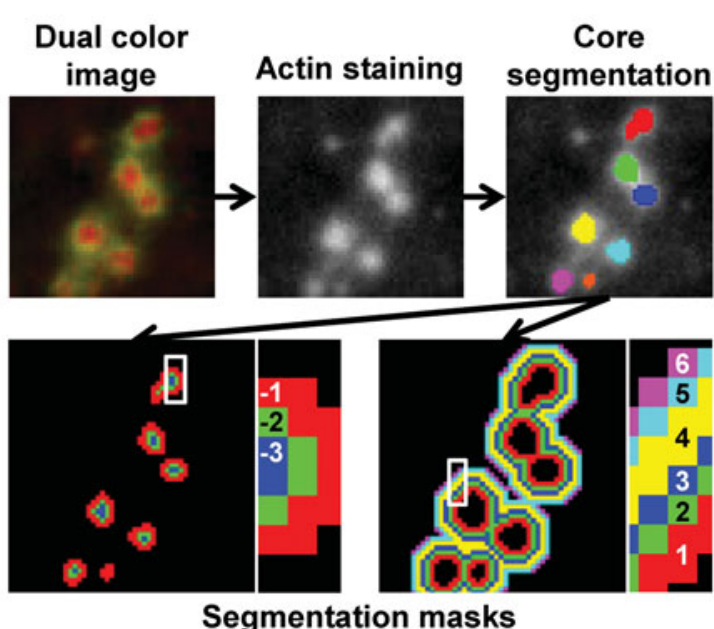

D

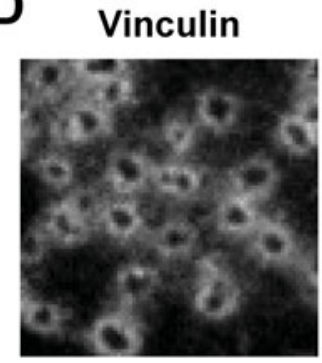

Paxillin
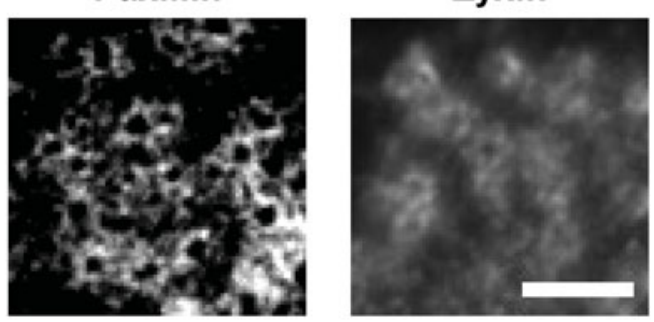

E
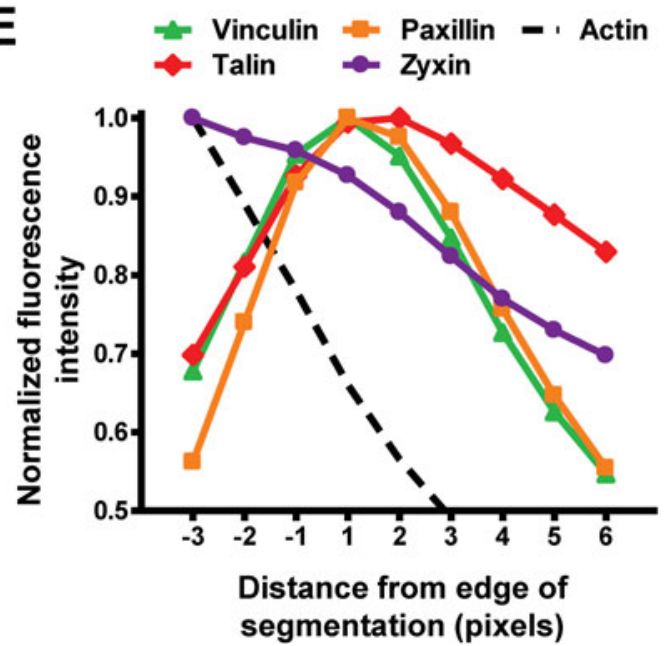

Figure 4. Podosome core and ring composition. DCs were seeded on glass coverslips, cells were left untreated (A, left), were treated for $30 \mathrm{~min}$ with $20 \mu \mathrm{M}$ blebbistatin (A, middle), or treated for 20 min with $2.5 \mu \mathrm{g} / \mathrm{mL}$ cytoD (A, right). Afterward they were fixed and labeled with Texas Red-conjugated phalloidin (A). For each condition median \pm interquartile range of the actin core intensity (left graph) and actin network intensity (right graph) for at least 800 podosomes is plotted in B. Data are normalized to the median intensity. Significant differences $(p<0.05)$ are indicated by asterisks. To measure podosome composition, DCs were seeded on glass coverslips, fixed and stained for F-actin (red) with (in green) either vinculin, talin, zyxin, or paxillin (C, upper left panel). The algorithm was applied to the F-actin image only (C, upper middle and left panel). Subsequently segmentation masks were created for all pixels in an image at a certain distance from the edge of a podosome core. This is done both inside (C, lower left panel) and outside $(\mathbf{C}$, lower right panel) the segmented areas. The numbers of the segmentation masks correspond to the $x$-axis in $\mathbf{E}$. Each color represents a separate segmentation mask. Immunolocalizations of vinculin, talin, zyxin, or paxillin are shown in $\mathbf{D}$. The mean fluorescence intensity (normalized to maximum) within each segmentation mask is plotted for all five proteins (E). The indicated distances ( $X$ axis) are the distances (in pixels) from the boundary of the podosome core segmentation, where negative values indicate areas inside and positive values indicate areas outside podosome core segmentations (C). For each condition five images, containing at least 1,120 podosomes were taken. Scale bars represent $3 \mu \mathrm{m}$.

The need for automated podosome detection partially results from the fact that it is very time-consuming to manually detect podosomes in an image, let alone large numbers of images derived from different experiments. For comparison, to identify all podosomes in the image shown in Supplementary Figure 1, experts needed on average $1 \mathrm{~h}$.
During this hour, the experts only pointed the positions of the podosome cores. By contrast, the algorithm was finished in $14 \mathrm{~s}$, and the output of the algorithm does not only contain the positions but also the boundaries of the core. In this way, intensity measurements of core components and, by manipulating the segmented areas, of ring components 
were very easy to implement. Another benefit of using an algorithm over manual identification is the fact that the algorithm is unbiased, while a researcher's eye is often preconceived.

We have tested the performance of the algorithm by comparing it to expert identifications of podosome cores, thereby demonstrating that the algorithm has a sensitivity of $73 \%$ with $16 \%$ false positives. Importantly, the interexpert overlap was also around $80 \%$ showing that there is no clear consensus among experts about which objects in the image should be defined as podosome cores. One could therefore argue that among the $16 \%$ false positives identified by the algorithm, there may be multiple objects that are actually podosome cores.

Since the algorithm can detect podosomes in live cell images of cells expressing an F-actin probe, it can be used to study podosome dynamics. In time-lapse movies the specificity of the detection will increase because unwanted objects can be disregarded if they are not consistent from frame to frame and appear in a few frames only. With a sampling frequency of a few frames per minute, podosomes are expected to be present in tens of consecutive frames before dissolving, thereby allowing the removal of segmentation artifacts, when they are present in only a few frames. The combination of our podosome detection algorithm with particle tracking algorithms such as described for receptor tracking (Jaqaman et al., 2008) will allow the study of podosome lifetimes as well as fission and fusion events in time-lapse movies. Another application of automated podosome detection could be to quantitatively distinguish between different meso-scale organizations of podosome clusters, such as the circumferential organization in osteoclasts (Destaing et al., 2003) or the alignment of podosomes in dendritic cells (van den Dries et al., 2012). Finally, this method might be useful for other image analysis purposes as well, where small features have to be separated from an uneven background resulting from a specific staining or localization of fluorescent probes.

It is challenging to visually estimate differences in podosome numbers, without manual counting, especially when large podosome clusters are present. This prompted us to evaluate the performance of the algorithm in quantifying podosome numbers, by applying it to images with decreasing podosome numbers per cell. The results of the algorithm were strikingly similar to the results manually obtained by three experts. The trend in the data is the same for the manually generated counts as for the algorithm output, except for a small underestimation in the samples with large numbers of podosomes. This is probably due to the fact that at higher densities podosomes that are very close together could be merged and considered as one feature or disregarded due to shape constraints. However, the advantage of performing the automatic analysis in minutes, rather than in hours as needed for manual counting, is the major advantage of this algorithm, which clearly represents an important tool for fast and accurate quantification of podosome numbers.
To demonstrate the applicability of the algorithm, we have used it to study podosome core and ring composition. We have established the effects of cytoD and blebb on the F-actin intensity in the podosome core and ring. By inhibiting actin polymerization, cytoD decreased F-actin intensity in both the podosome core and actin network surrounding the core. By contrast, myosin II inhibition resulted in an increase in F-actin intensity in both core and actin network. Because automated detection can very efficiently measure thousands of podosomes, small but relevant differences in intensity levels could be detected for different drug treatments with sufficient statistical power.

We also used the algorithm to assess the localization of podosome ring components with respect to the actin core. Hereby, we showed that vinculin, talin, paxillin, and zyxin are differentially distributed around the podosome cores. Talin displayed a homogeneous distribution between the cores, whereas vinculin, paxillin, and zyxin are present as a clear ring around the core. Interestingly, the vinculin and paxillin rings have a diameter larger than the core while the zyxin ring is smaller. This type of large-scale analysis of the localization of podosome components can clearly contribute to the understanding of podosome structure and the function of its components. Collectively, we demonstrate that the algorithm we developed can readily be used to study podosome numbers and the composition of the podosome actin core, the actin network, and ring components, providing a new tool to identify yet unknown changes in podosome composition.

\section{CONCLUSIONS/SUMMARY}

In summary, the proposed method automatically identifies podosome cores in fluorescence image of cells with high sensitivity and accuracy compared to expert identifications. It thereby provides a useful tool to study podosome numbers and podosome core and ring composition in a fast, quantitative, automated, and unbiased way.

\section{ACKNOWLEDGMENTS}

The authors thank Ben Joosten for his contribution as an expert in the manual analysis of podosome images, Michael Sixt for the kind gift of the LifeAct-GFP construct, and Jack Fransen for critically reading the manuscript. All microscopy experiments were conducted at the Microscopic Imaging Centre of the Nijmegen Centre for Molecular Life Sciences. This research was supported by EU grants BIOLIGHT-TOUCH (028781) and Immunanomap (MRTN-CT2006-035946) awarded to C.G.F. and an EU grant Nanovista (ICT-2011.3.5/288263) and a Young investigator Grant from the Human Frontier Science Program (RGY0074/2008) awarded to A.C. M.B.M.M. is supported by the REMEDI (HEALTH-F5-2009-242276) grant. A.C. is the recipient of a Meervoud grant (836.09.002), and C.G.F. was awarded with a Spinoza prize, both from The Netherlands Organization for Scientific Research (NWO). 


\section{REFERENCES}

Block, M.R., Badowski, C., Millon-Fremillon, A., Bouvard, D., Bouin, A.P., Faurobert, E., Gerber-Scokaert, D., Planus, E. \& Albiges-Rizo, C. (2008). Podosome-type adhesions and focal adhesions, so alike yet so different. Eur J Cell Biol 87 (8-9), 491-506.

Blouw, B., Seals, D.F., Pass, I., Diaz, B. \& Courtneidge, S.A. (2008). A role for the podosome/invadopodia scaffold protein Tks5 in tumor growth in vivo. Eur J Cell Biol 87 (8-9), 555-567.

Destaing, O., Saltel, F., Geminard, J.C., Jurdic, P. \& Bard, F. (2003). Podosomes display actin turnover and dynamic selforganization in osteoclasts expressing actin-green fluorescent protein. Mol Biol Cell 14(2), 407-416.

de Vries, I.J., Eggert, A.A., Scharenborg, N.M., Vissers, J.L., Lesterhuis, W.J., Boerman, O.C., Punt, C.J., Adema, G.J. \& FIgDor, C.G. (2002). Phenotypical and functional characterization of clinical grade dendritic cells. J Immunother 25(5), 429-438.

Evans, J.G., Correia, I., Krasavina, O., Watson, N. \& MatsuDAIRA, P. (2003). Macrophage podosomes assemble at the leading lamella by growth and fragmentation. J Cell Biol 161(4), 697-705.

Feret, L.R. (1931). La grosseur des grains. Zurich: Assoc. Intern. Essais Math. 2D.

Gawden-Bone, C., Zhou, Z., King, E., Prescott, A., Watts, C. \& LucocQ, J. (2010). Dendritic cell podosomes are protrusive and invade the extracellular matrix using metalloproteinase MMP14. J Cell Sci 123(Pt 9), 1427-1437.

Jaqaman, K., Loerke, D., Mettlen, M., Kuwata, H., Grinstein, S., Schmid, S.L. \& Danuser, G. (2008). Robust single-particle tracking in live-cell time-lapse sequences. Nat Methods 5(8), 695-702.

Jiang, S., Zhou, X.B., Kirchhausen, T. \& Wong, S.T.C. (2007). Detection of molecular particles in live cells via machine learning. Cytometry Part A 71A(8), 563-575.

Kalaidzidis, Y. (2007). Intracellular objects tracking. Eur J Cell Biol 86(9), 569-578.

Kimori, Y., BabA, N. \& Morone, N. (2010). Extended morphological processing: A practical method for automatic spot detection of biological markers from microscopic images. $B M C$ Bioinformatics 11, 373.

Linder, S. (2007). The matrix corroded: Podosomes and invadopodia in extracellular matrix degradation. Trends Cell Biol 17(3), 107-117.

Luxenburg, C., Geblinger, D., Klein, E., Anderson, K., HAnein, D., Geiger, B. \& Addadi, L. (2007). The architecture of the adhesive apparatus of cultured osteoclasts: From podosome formation to sealing zone assembly. PLoS One 2(1), e179.

Luxenburg, C., Winograd-Katz, S., Addadi, L. \& Geiger, B. (2012). Involvement of actin polymerization in podosome dynamics. J Cell Sci 125(Pt 7), 1666-1672.

Mukhopadhyay, U.K., Eves, R., Jia, L., Mooney, P. \& MaK, A.S. (2009). p53 suppresses Src-induced podosome and rosette formation and cellular invasiveness through the upregulation of caldesmon. Mol Cell Biol 29(11), 3088-3098.

Murphy, D.A. \& Courtneidge, S.A. (2011). The 'ins' and 'outs' of podosomes and invadopodia: Characteristics, formation and function. Nat Rev Mol Cell Biol 12(7), 413-426.

Niblack, W. (1986). An Introduction to Digital Image Processing. Englewood Cliffs, N.J: Prentice-Hall.

Olivo-Marin, J.C. (2002). Extraction of spots in biological images using multiscale products. Pattern Recogn 35(9), 1989-1996.

Ridler, T.W. \& Calvard, S. (1978). Picture thresholding using an iterative selection method. IEEE Trans Syst Man Cybern 8(8), 630-632.

Smal, I., Loog, M., Niessen, W. \& Meijering, E. (2010). Quantitative comparison of spot detection methods in fluorescence microscopy. IEEE Trans Med Imaging 29(2), 282-301.

Thurner, B., Roder, C., Dieckmann, D., Heuer, M., Kruse, M., Glaser, A., Keikavoussi, P., Kampgen, E., Bender, A. \& Schuler, G. (1999). Generation of large numbers of fully mature and stable dendritic cells from leukapheresis products for clinical application. J Immunol Methods 223(1), 1-15.

van den Dries, K., van Helden, S.F., Riet, J., Diez-Ahedo, R., Manzo, C., Oud, M.M., van Leeuwen, F.N., Brock, R., GarciaParajo, M.F., Cambi, A. \& Figdor, C.G. (2012). Geometry sensing by dendritic cells dictates spatial organization and PGE(2)-induced dissolution of podosomes. Cell Mol Life Sci 69(11), 1889-1901.

van Helden, S.F., Krooshoop, D.J., Broers, K.C., Raymakers, R.A., Figdor, C.G. \& van LeEuwen, F.N. (2006). A critical role for prostaglandin E2 in podosome dissolution and induction of high-speed migration during dendritic cell maturation. J Immunol 177(3), 1567-1574.

van Helden, S.F., Oud, M.M., Joosten, B., Peterse, N., Figdor, C.G. \& van Leeuwen, F.N. (2008). PGE2-mediated podosome loss in dendritic cells is dependent on actomyosin contraction downstream of the RhoA-Rho-kinase axis. J Cell Sci 121(Pt 7), 1096-1106.

VinCENT, L. \& SoILle, P. (1991). Watersheds in digital spaces-An efficient algorithm based on immersion simulations. IEEE Trans Pattern Anal Mach Intell 13(6), 583-598. 